(NASA-TM-111113)

(NIPS-95-05511) OPTIMIZATION OF MOISTURE CONTENT FOR WHEAT SEEDLING

N96-13101 GERMINATION IN A CELLULOSE ACETATE MEOIUM FOR A SPACE FLIGHT

EXPERIMENT (NASA. KennedY SPace

Unclas

Center) $10 \mathrm{p}$

$63 / 51 \quad 0072790$ 


\title{
OPTIMIZATION OF MOISTURE CONTENT FOR WHEAT SEEDLING GERMINATION IN A CELLULOSE ACETATE MEDIUM FOR A SPACE FLIGHT EXPERIMENT
}

\author{
Corinne F. Johnson ${ }^{1}$, Thomas W. Dreschel ${ }^{1}$, Christopher S. Brown ${ }^{1}$, and Raymond M. \\ Wheeler ${ }^{2}$
}

IThe Bionetics Corporation, Code BIO-3, Kennedy Space Center, FL 32899 USA 2National Aeronautics and Space Administration, Code MD-RES, Kennedy Space Center, FL 32899 USA

\begin{abstract}
The Porous Tube Plant Nutrient Delivery System (PTPNDS), a hydrophilic, microporous ceramic tube hydroponic system designed for microgravity, will be tested in a middeck locker of the Space Shuttle. The flight experiment will focus on hardware operation and assess its ability to support seed germination and early seedling growth in microgravity. The water controlling system of the PTPNDS hardware has been successfully tested during the parabolic flight of the $\mathrm{KC}-135$. One challenge to the development of the spaceflight experiment was to devise a method of holding seeds to the cylindrical porous tube. The seedholder must provide water and air to the seed, absorb water from the porous tube, withstand sterilization, provide a clear path for shoots and roots to emerge, and be composed of flight qualified materials. In preparation for the flight experiment, a wheat seed-holder has been designed that utilizes a cellulose acetate plug to facilitate imbibition and to hold the wheat seeds in contact with the porous tube in the correct orientation during the vibration of launch and the microgravity environment of orbit. Germination and growth studies with wheat at a range of temperatures showed that optimal moisture was $78 \%$ (by weight) in the cellulose acetate seed holders. These and other design considerations are discussed.
\end{abstract}

\section{INTRODUCTION}

The Porous Tube Plant Nutrient Delivery System (PTPNDS), developed at NASA's Kennedy Space Center, is a hydroponic system designed to deliver water and nutrients to plants in microgravity /1-3/. A Space Shuttle middeck locker experiment to test the system in microgravity is tentatively scheduled for flight in early 1996 designated as the Microgravity Plant Nutrient Experiment (MPNE). This paper describes the design of the seed-holding apparatus, biocompatibility testing and moisture optimization studies. 


\section{TEST HARDWARE DESCRIPTION}

Water and nutrients are circulated through a hollow porous ceramic tube (Part \# 46826, Osmonics, Minnetonka, Minnesota)* and they reach the outer tube surface by capillary action through the tube's $0.7 \mu \mathrm{m}$ pores. An infrared sensor measures tube surface wetness by monitoring changes in infrared reflectance on the tube surface. The infrared sensor relays information to a controlling computer, which activates a steppermotor controlled syringe to adjust the amount of water in the fluid circuit to maintain the desired tube wetness. The infrared sensor and controlling system successfully controlled tube surface wetness during KC-135 flight tests /4/.

Mounted to the surface of each of three 23-cm long tubes are seed-holding cassettes (Fig. 1). The seed cassettes are designed to provide a germination medium and to maintain seed position and orientation during the vibration of launch and the microgravity environment of orbit. Each seed cassette has 10 wells with a cylinder-shaped plug of cellulose acetate material (conventionally used as a pipette filter) (Rainin Instrument Company \# 23534B, Woburn, Massachusetts)* placed in each well. The cellulose acetate filters are flight qualified, and were flown on the Space Shuttle in 1993 in the CHROMEX IV middeck locker experiment /5/. The seeds are held by friction in the cellulose acetate plugs, which are in contact with the tube surface. Wheat (Triticum aestivum cv Yecora rojo) and lettuce seeds (Lactuca sativa cv Parris Island Cos) will be used in the flight experiment. Seeds are oriented so that as the seedlings emerge, shoots extend away from the tube and roots grow through the germination plugs to contact the porous tube surface below. In ground studies, the roots typically emerge from the plug and grow along the tube surface by 4 days after imbibition. The seed cassette extends to cover the outer surface of the tube without contacting the tube. Prototype seed cassettes have included a plastic sheet which wraps around the porous tube and is held in place with nylon hook-and-loop tape (Velcro \#191241 \& 191, Velcro USA Manchester, New Hampshire)*. This tube covering maintains a dark, humid environment for growing plant roots. Once the roots reach the tube surface, the cellulose acetate plug serves primarily as a stabilizing structure to maintain seedling position for the duration of the experiment. Ground studies were conducted to 1) determine the optimal moisture content for wheat seedling germination in the cellulose acetate plugs, 2) evaluate uniformity of the cellulose acetate plugs and 3) determine the relationship between tube surface wetness and cellulose acetate plug moisture content.

\section{MATERIALS AND METHODS}

\section{Evaluation of Uniformity of Cellulose Acetate Plugs}

\section{Effect of orientation on plug moisture content measured on a static water system. A porous tube was} connected to a static water reservoir at a height of $15 \mathrm{~cm}$ above the water line. A syringe was used to remove air bubbles from the tube. Absorbent cellulose acetate filters were cut in half with scissors to form two plugs, and the plugs were then weighed. The plugs were positioned on the porous tabe, and held in place by the seed cassette. Half of the plugs were oriented with the smooth (factory-cut) side against the porous tube 
and the other half with the scissor-cut side against the porous tubes. Plugs were allowed to absorb water and stabilize for one hour and were then removed from the tube and re-weighed. Percent moisture was calculated. The test was repeated three times, with a total sample size of 21 plugs for each orientation.

Fig. 1. MPNE seed holding cassette, exploded side view showing cellulose acetate plugs and seeds as mounted to porous ceramic tube.

Effect of orientation on wheat growth measured in a circulating water system. Four porous tubes were mounted to a manifold connecting the tubes to a fluid circuit. Water was circulated through the porous tubes by a diaphragm pump. Seedlings were grown for 7 days under high-pressure sodium lamps $\left(500 \mu \mathrm{mol} \mathrm{m}^{-2}\right.$ $\mathrm{s}^{-1}$ ), at a temperature of $23^{\circ} \mathrm{C}$. The growth experiment was performed twice, with a total sample size of 27 seedlings for each plug orientation.

\section{Cellulose Acetate Plug's Moisture Content: Effect on Wheat and Lettuce Germination}

Cellulose acetate plugs were cut in half and one seed was planted in each plug. Seeded plugs were placed standing in 30-ml covered glass jars. Five plugs were placed in each jar, with four jars per moisturetreatment for each seed type. Ten different amounts of water (from 0.1 to $1.0 \mathrm{ml}$, in $100 \mu l$ increments) were pipetted into each plug. Wheat seedlings were grown for 5 days and lettuce seedlings were grown for 10 days. Experiments were conducted under high-pressure sodium lamps $\left(500 \mu \mathrm{mol} \mathrm{m} \mathrm{m}^{-2} \mathrm{~s}^{-1}\right)$, at a temperature of $23^{\circ} \mathrm{C}$. Germination and seedling growth were measured. Each experiment was performed twice, for a total sample size of 40 seedlings per each of the ten moisture-level treatments. 
Cellulose acetate plug moisture and tube wetness in a circulating water system. Cellulose acetate filters were cut in half with scissors and weighed. The plugs were positioned on the porous tubes with the factory-cut side facing the tube, and the MPNE controlling computer was set to one tube-surface-wetness set point. Plugs absorbed water from the tubes overnight and were then removed from the tubes and re-weighed. The difference between starting and ending weight was the weight of water absorbed and maintained in each plug. This procedure was repeated for two consecutively higher tube-surface-wetness set points and the experiment was repeated three times for each set point.

\section{RESULTS}

Evaluation of Uniformity of Cellulose Acetate Plugs

Effect of orientation on plug moisture content measured on a static water system. A t-test was performed to compare average moisture content between plugs with the factory cut or scissor cut side against the tube surface and showed no significant difference (TABLE 1).

TABLE 1 Comparison of average percent moisture (by weight) of cellulose acetate plugs in two orientations $(\mathrm{n}=21),(\mathrm{P}<.005)$.

Effect of orientation on wheat growth measured in a circulating water system. A t-test performed to analyze measurements of seedling height and coleoptile length demonstrated that seedling growth was not significantly affected by plug orientation $(P<.005)$ (TABLE 2$)$.

\section{Cellulose Acetate Plug's Moisture Content: Effect on Wheat and Lettuce Germination}

Moisture content of the cellulose acetate plugs affected germination rates of wheat and lettuce seeds (Fig. 2.) The best germination was obtained in filters containing 0.2 to $0.4 \mathrm{ml}$ of water for wheat and 0.4 to $0.6 \mathrm{ml}$ for lettuce. Plugs became saturated at $0.6 \mathrm{ml}$. Wheat germination fell sharply after $0.5 \mathrm{ml}$. The small lettuce seeds located at the top of the filter did not appear to be as adversely effected by the higher moisture levels, although lettuce germination declined after $0.8 \mathrm{ml}$ per plug. 
TABLE 2 Comparison of seedling height and coleoptile length of wheat seedlings tested in two orientations for 27 measurements for each orientation.

Fig. 2. Germination curves for wheat and lettuce as a function of plug moisture content. Each point represents data from 40 seeds.

\section{Relationship between Tube Surface Moisture and Plug Moisture Content}

Plug moisture content increased with increasing the tube wetness set-point. Over the range of moistures tested, the response was linear (Fig. 3). The correlation ( $r$ ) to the line of best fit $(y=597-988 x$ ) was $r=$ 0.986. Each point on the graph below represents data from three experiments. Bars on the graph show standard deviation. 
Fig. 3. Plug moisture content response curve as a function of increasing (computercontrolled) tube-wetness set points. Set point units expressed as voltage.

\section{DISCUSSION and CONCLUSIONS}

In designing and optimizing a seed holding mechanism for a space flight experiment, several issues have been addressed. A cellulose acetate plug was chosen to hold the seeds while providing a germination medium to the seeds. In addition to being previously flown on the Space Shuttle, the material under consideration has performed well during ground studies for MPNE. The cellulose acetate plugs provide a uniform supply of water to the germinating seeds even after the slight compaction caused by cutting the plugs with scissors. The plugs respond to changes in the porous tube surface wetness so that moisture levels in the plugs can be controlled by computer. A moisture level of 0.3 to $0.4 \mathrm{ml}$ per plug will be used for the space flight experiment. This is equivalent to approximately $78 \%$ (by weight) moisture content in the plug and yields excellent germination ( $>90 \%$ ) in both lettuce and wheat. A significant drop in wheat germination occurred for amounts greater than $0.5 \mathrm{ml}$ per plug and considerations must be made for the change in behavior of fluids in microgravity /6-8/ where there is no gravitational component in determining water potential and distribution. While $0.4 \mathrm{ml}$ per plug may be optimal for germination in a $1 \mathrm{~g}$ field, the fluid may not be distributed in the same way within the plug in mictogravity $19 \%$. However, spaceflight soil-moisture content experiments on sunflower seedlings, performed in preparation for the Spacelab 1 HEFLEX experiment, showed no significant differences in germination or growth compared to earth-grown seedlings $/ 10 \%$. Water distribution in microgravity may be different for different culture media. Therefore, in order to maintain a high germination potential for the flight seed in a cellulose acetate medium, a conservative amount of water ( 0.3 to $0.4 \mathrm{ml}$ ) will be supplied to the germinating seeds. 
*The use of a brand name does not imply endorsement of the product by The Bionetics Corporation or NASA.

\section{REFERENCES}

1. T.W. Dreschel, Plant nutrient delivery system having a porous tubular member. Patent \# 4,926,585. United States Patent and Trademark Office, Washington, D.C. (1990).

2. T.W. Dreschel, Tubular membrane plant-growth unit. NASA Tech Briefs 16(2):113.(1992).

3. T.W. Dreschel and Brown, C.S., Water conserving plant growth system. NASA Tech Briefs 17(1):89. (1993).

4. T.W. Dreschel ${ }_{2}$ Carlson, C.W., Wells, H.W., Anderson, K.F., Knott, W.M. and Munsey, W. Physical testing for the microgravity plant nutrient experiment. ASAE Paper No, 23-4007.(1993).

5. A.G. Heyenga and Lewis, N.G., A solid state nutrient support system for long term plant culture in microgravity conditions, (manuscript in preparation) (1994).

6. P.J. Sell, Maisch, E., and Siekmann, J. Fluid transport in capillary systems under microgravity. Acta Astronautica, (11), \# 9, 577.(1983).

7. L.E. Scriven and Sternling, C.V., The Marangoni effects. Nature, (187), 186.(1960).

8. T.P. Sheahen, Physical principles of microgravity research. Report to NASA Headquarters Office of Space Commercialization. NASA order \#W-15596. (1986).

9. H.G. Levine, private communication (1993).

10. A.H. Brown and D.K. Chapman, A test to verify the biocompatibility of a method for plant culture in a microgravity environment. Annals of Botany (54) Supplement 3, 19 (1984). 
TABLE 1 Comparison of average percent moisture (by weight) of cellulose acetate plugs in two orientations $(n=21)$ shows no significant difference $(P<.005)$.

\begin{tabular}{ccc}
\hline $\begin{array}{c}\text { Side of Plug } \\
\text { Facing Tube }\end{array}$ & $\begin{array}{c}\text { Average \% } \\
\text { Moisture }\end{array}$ & $\begin{array}{c}\text { Standard } \\
\text { Error }\end{array}$ \\
\hline $\begin{array}{c}\text { Factory-cut Side } \\
-\end{array}$ & 79.3 & \pm 0.02 \\
Scissor-cut Side & 79.0 & \pm 0.02 \\
\hline
\end{tabular}

TABLE2 Comparison of seedling height and coleoptile length of wheat seedlings tested in two orientations for 27 measurements for each orientation shows no significant difference $(P<.005)$.

\begin{tabular}{lcccc}
\hline $\begin{array}{l}\text { Side of Plug } \\
\text { Facing Tube }\end{array}$ & $\begin{array}{c}\text { Height } \\
(\mathrm{mm})\end{array}$ & $\begin{array}{c}\text { Standard } \\
\text { Enror }\end{array}$ & $\begin{array}{c}\text { Coleoptile } \\
\text { Length }(\mathrm{mm})\end{array}$ & $\begin{array}{c}\text { Standard } \\
\text { Error }\end{array}$ \\
\hline & 81.4 & 0.47 & 15.0 & \pm 0.09 \\
Factory-cut Side & 79.2 & 0.52 & 14.7 & \pm 0.08 \\
\hline
\end{tabular}



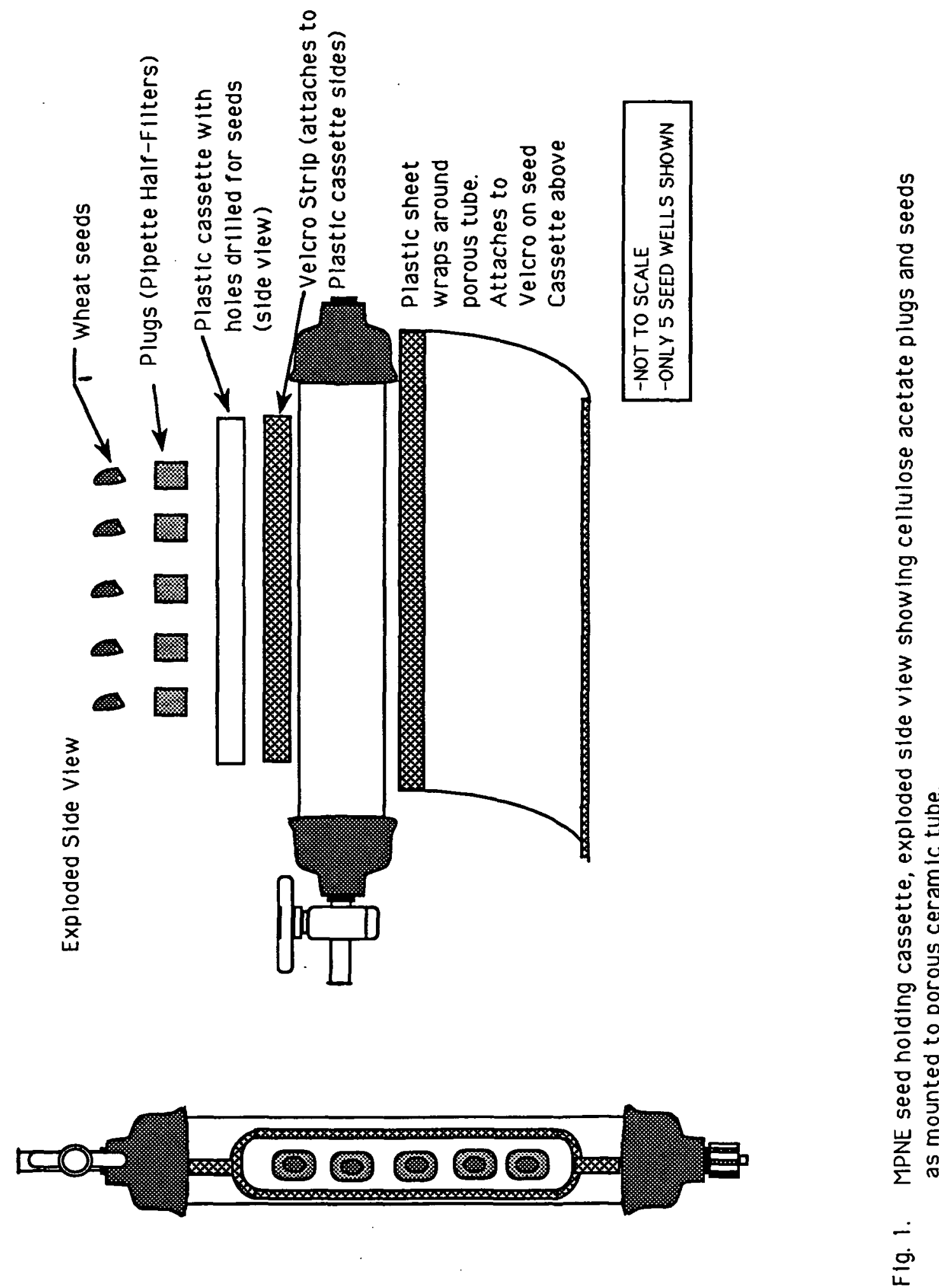

$\frac{3}{2}$ 


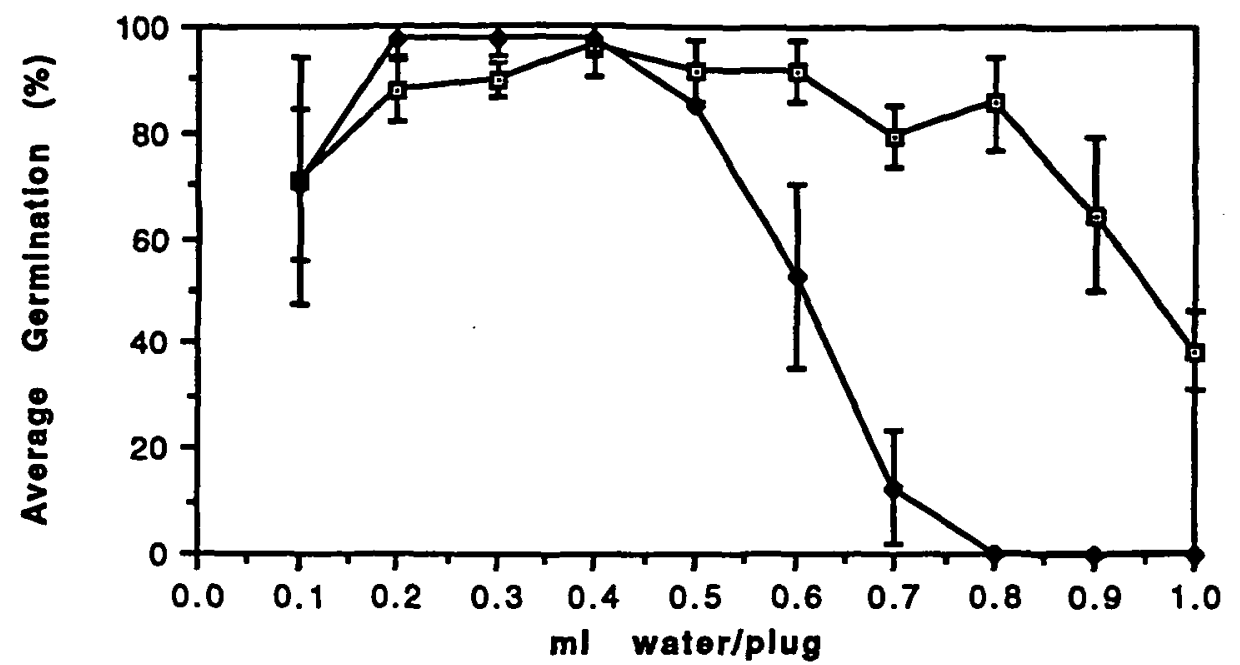

$\longrightarrow$ Average Lettuce Germination (\%) ـ

Fig. 2. Germination curves for wheat and lettuce as a function of plug moisture content. Each point represents data from $\mathbf{4 0}$ seeds.

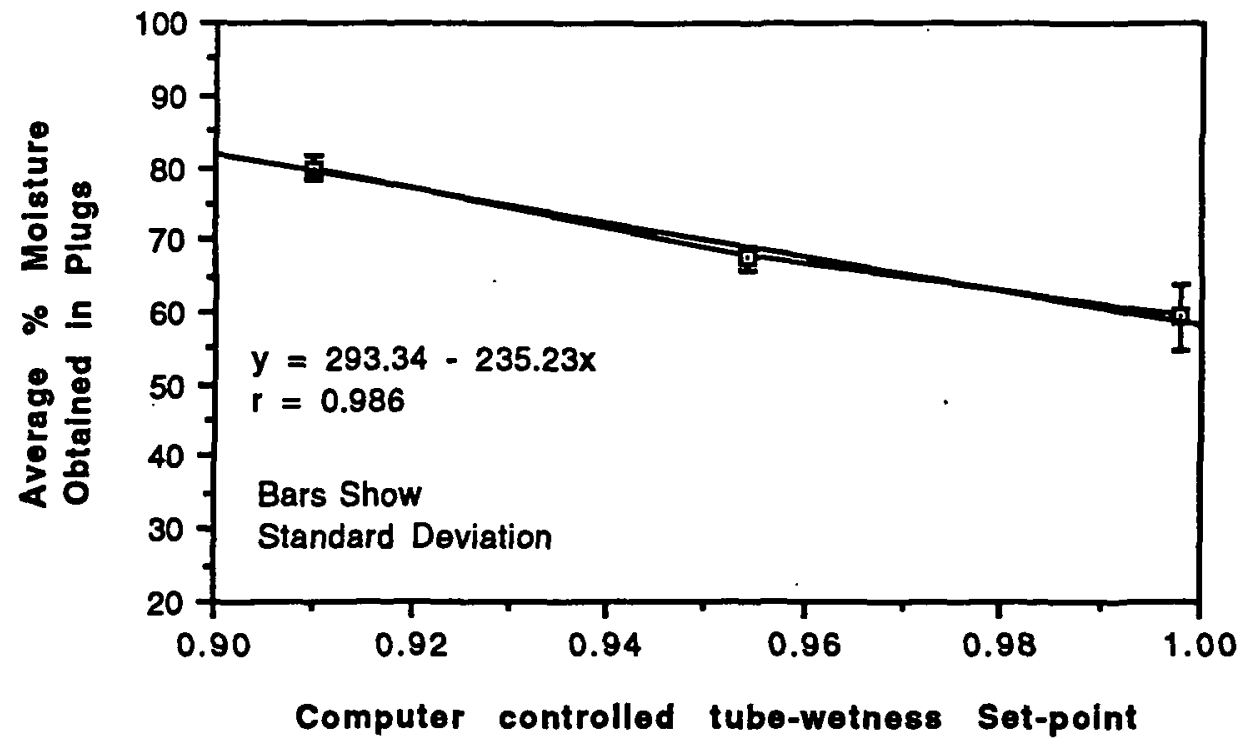

Fig. 3. Plug moisture content response curve as a function of increqaseing (computercontrolled) tube-wetness set points. Set point units expressed as voltage. 